\title{
EXPLICIT QUANTIZATION OF THE KEPLER MANIFOLD
}

\author{
ROBERT J. BLATTNER ${ }^{1}$ AND JOSEPH A. WOLF ${ }^{2}$
}

\begin{abstract}
Any representation $\pi$ of $\operatorname{SO}(2,4)$ quantizing the Kepler manifold has the same lowest highest weight as the representation $\nu_{0}$ in the Sternberg-Wolf description of the $U(2,2)$-restriction of the metaplectic representation of $\mathrm{Sp}(4 ; \mathbf{R})$. Hence, modulo covering groups, $\pi$ is unitarily equivalent to $\nu_{0}$.
\end{abstract}

0. Introduction. The Kepler manifold $T^{+}\left(S^{3}\right)$ is the cotangent bundle of the 3-sphere, minus the zero section, with the symplectic structure induced by that of the cotangent bundle. It is a Hamiltonian symplectic homogeneous space of the conformal group $\operatorname{SO}(2,4)$ (cf. [11]). The action of $\operatorname{SO}(2,4)$ on $T^{+}\left(S^{3}\right)$ has been quantized by various authors ([3], [6], [7], [10], [11]) to give an irreducible unitary representation $\pi$ of $\operatorname{SO}(2,4)$ on $L^{2}\left(S^{3}\right)$. However, these constructions of $\pi$ suffer from being either ad hoc or else arrived at by a limiting procedure. Here, we give an explicit identification of $\pi$ within the framework of the metaplectic representation.

In this note we show that $\pi$ is essentially unitarily equivalent to a certain representation $\nu_{0}$ of the 2 -sheeted cover $\operatorname{SU}(2,2)$ of $\operatorname{SO}(2,4)$, described in [9]. It was noted in [12] that the coadjoint orbit of $\operatorname{SU}(2,2)$ which corresponds to $\nu_{0}$ under the moment map [4] is the coadjoint orbit of $\operatorname{SO}(2,4)$ symplectomorphic to $T^{+}\left(S^{3}\right)$, which of course suggests, but does not prove, that $\pi=\nu_{0}$. Here we prove this equivalence by examining restrictions to a maximal compact subgroup of $\mathrm{SU}(2,2)$. The interpretation of this result in terms of geometric quantization will be the subject of another paper.

1. The representations $\pi$ and $\pi_{1}$. Rawnsley [8] and Blattner [1] have discussed two positive polarizations of the symplectic manifold $T^{+}\left(S^{3}\right)$ and their corresponding Hilbert spaces. The first polarization $F$ is just the cotangent fibration, and the associated Hilbert space $\mathcal{H}_{F}$ is naturally isomorphic to $L^{2}\left(S^{3}\right)$. The second polarization, $G$, is obtained as follows: Identify $T^{+}\left(S^{3}\right)$ with

$$
\left\{(e, x) \in \mathbf{R}^{4} \times \mathbf{R}^{4}: e \cdot e=1, e \cdot x=0, x \neq 0\right\} .
$$

Send $(e, x)$ to $|x| e+i x \in \mathbf{C}^{4}$. The image is

$$
X=\left\{z \in \mathbf{C}^{4}: z \cdot z=0, z \neq 0\right\},
$$

Received by the editors September 27, 1978.

AMS (MOS) subject classifications (1970). Primary 22E45, 22E70; Secondary 53C15, 81A54.

${ }^{1}$ Research partially supported by NSF Grant MCS 78-01332 and MCS 75-17621.

${ }^{2}$ Research partially supported by NSF Grant MCS 76-01692.

(C) 1979 American Mathematical Society 0002-9939/79/0000-0480/\$02.25 
$X$ is then a Kähler manifold with Kähler form

$$
-2^{-5 / 2} i d\left(|z|^{-1}\{z \cdot d \bar{z}-\bar{z} \cdot d z\}\right) \text {. }
$$

This structure defines $G$. The associated Hilbert space $\mathcal{H}_{G}$ consists of holomorphic functions on $X$ square integrable with respect to the measure

$$
\exp (-4 \pi|x|) 2^{5 / 2}|x|^{1 / 2} \gamma
$$

where $\gamma$ is the Liouville measure on $X$.

There is no positive polarization of $T^{+}\left(S^{3}\right)$ stable under $\operatorname{SO}(2,4)$ [11]. However $F$ is $\mathrm{SO}(1,4)$ stable and $G$ is $\mathrm{SO}(2) \times \mathrm{SO}(4)$ stable, and hence geometric quantization [2] provides unitary representations $\pi_{F}$ of $\operatorname{SO}(1,4)$ on $\mathcal{H}_{F}$ and $\pi_{G}$ of $\mathrm{SO}(2) \times \mathrm{SO}(4)$ on $\mathcal{H}_{G}$. Moreover, the half form pairing of $\mathcal{H}_{F}$ with $\mathcal{K}_{G}$ (see [8]) gives a bounded nonunitary operator $T$ of $\mathcal{K}_{F}$ onto $\mathcal{K}_{G}$, with bounded inverse, which intertwines $\left.\pi_{F}\right|_{\text {SO(4) }}$ and $\left.\pi_{G}\right|_{\text {SO(4) }}$.

For our purposes $\pi$ will be any irreducible unitary representation of $\mathrm{SO}(2,4)$ such that $\left.\pi\right|_{\mathrm{SO}(1,4)} \cong \pi_{F}$ and $\left.\pi\right|_{\mathrm{SO}(2) \times \operatorname{SO}(4)} \cong \pi_{G}$.

As usual, the indefinite unitary group $U(2,2)=\left\{g \in \mathbf{C}^{4 \times 4}: g h g^{*}=h\right\}$, where

$$
h=\left(\begin{array}{cc}
I_{2} & 0 \\
0 & -I_{2}
\end{array}\right),
$$

and $\mathbf{S U}(2,2)=\{g \in \mathbf{U}(2,2)$ : $\operatorname{det} g=1\}$. Now $\wedge^{2}\left(\mathbf{C}^{4}\right)=\mathbf{C}^{6}$ has a real form $\mathbf{R}^{6}$ invariant under $\left\{\wedge^{2}(g): g \in \operatorname{SU}(2,2)\right\}$. This action of $\operatorname{SU}(2,2)$ on $\mathbf{R}^{6}$ preserves a nondegenerate quadratic form of signature $(2,4)$. In this way we get a homomorphism $\alpha$ : $\mathrm{SU}(2,2) \rightarrow \mathrm{SO}(2,4)$, where $\alpha(g)=\left.\wedge^{2}(g)\right|_{\mathbf{R}^{6}}$, and this $\alpha$ is in fact a double covering. Letting $\pi_{1}=\pi \circ \alpha$, we obtain an irreducible unitary representation of $\operatorname{SU}(2,2)$.

2. The representation $\nu_{0}$. Fix a nondegenerate antisymmetric bilinear form $\{u, v\}$ on $\mathbf{R}^{8}$. The symplectic group $\operatorname{Sp}(4 ; \mathbf{R})$ is the automorphism group of $\left(\mathbf{R}^{8},\{\cdot, \cdot\}\right)$. If $u, v \in \mathbf{R}^{8}$, then $\xi_{u, v}: x \mapsto \frac{1}{2}(\{u, x\} v+\{v, x\} u)$ belongs to the Lie algebra $\mathfrak{s p}(4 ; \mathbf{R})$. Fix a basis $p_{1}, \ldots, p_{4}, q_{1}, \ldots, q_{4}$ of $\mathbf{R}^{8}$ with $\left\{p_{i}, p_{j}\right\}=$ $\left\{q_{i}, q_{j}\right\}=0$ and $\left\{p_{j}, q_{k}\right\}=\delta_{j k}$. Then $\mathfrak{i p}(4 ; \mathbf{R})$ has basis

$$
\xi_{a, b}=\xi_{p_{a}, p_{b}}, \quad \xi_{a, b}^{\prime}=\xi_{p_{a}, q_{b}}, \quad \xi_{a, b}^{\prime \prime}=\xi_{q_{a}, q_{b}} .
$$

Let $\lambda$ be Lebesgue measure on $C^{4}$. We have a Hilbert space

$$
\mathcal{K}=\left\{f: \mathbf{C}^{4} \rightarrow \mathbf{C} \text { holomorphic: } \int|f(z)|^{2} \exp \left(-|z|^{2}\right) d \lambda<\infty\right\}
$$

with inner product

$$
\left\langle f_{1}, f_{2}\right\rangle=\pi^{-4} \int f_{1}(z) \overline{f_{2}(z)} \exp \left(-|z|^{2}\right) d \lambda .
$$

In multi-index notation $z^{n}=z_{1}^{n_{1}} \cdots z_{4}^{n_{4}}, n !=n_{1} ! \cdots n_{4} !$, where $n=$ $\left(n_{1}, \ldots, n_{4}\right)$, the $\varphi_{n}(z)=z^{n} / \sqrt{n !}$ form an orthonormal basis of $\mathcal{H}$.

The metaplectic group $\mathrm{Mp}(4 ; \mathbf{R})$ is the two-sheeted covering group of 
$\mathrm{Sp}(4 ; \mathbf{R})$. It has a unitary representation $\mu$ on $\mathcal{K}$, called the metaplectic representation, specified by

$$
\begin{aligned}
& d \mu\left(\xi_{a, b}\right)=-\frac{i}{2}\left(\partial_{a} \partial_{b}-z_{a} \partial_{a}-z_{b} \partial_{a}+z_{a} z_{b}-\delta_{a, b}\right), \\
& d \mu\left(\xi_{a, b}^{\prime}\right)=\frac{1}{2}\left(\partial_{a} \partial_{b}-z_{a} \partial_{b}+z_{b} \partial_{a}-z_{a} z_{b}\right), \\
& d \mu\left(\xi_{a, b}^{\prime \prime}\right)=\frac{i}{2}\left(\partial_{a} \partial_{b}+z_{a} \partial_{b}+z_{b} \partial_{a}+\delta_{a, b}\right),
\end{aligned}
$$

where $\partial_{a}=\partial / \partial z_{a}$ (see [9]).

Now $U(2,2)$ is naturally isomorphic to the subgroup of $\operatorname{Sp}(4 ; \mathbf{R})$ with Lie algebra spanned by the

$$
\begin{array}{ll}
\xi_{a, b}+\xi_{a, b}^{\prime \prime}, & 1 \leqslant a \leqslant b \leqslant 2 \text { or } 3 \leqslant a \leqslant b \leqslant 4, \\
\xi_{a, b}^{\prime}-\xi_{b, a}^{\prime}, & (a, b)=(1,2) \text { or }(3,4), \\
\xi_{a, b}-\xi_{a, b}^{\prime \prime}, & 1 \leqslant a \leqslant 2 \text { and } 3 \leqslant b \leqslant 4, \\
\xi_{a, b}^{\prime}+\xi_{b, a}^{\prime}, & 1 \leqslant a \leqslant 2 \text { and } 3 \leqslant b \leqslant 4 .
\end{array}
$$

Moreover

$$
\begin{aligned}
& d \mu\left(\xi_{a, b}+\xi_{a, b}^{\prime \prime}\right)=i\left(z_{a} \partial_{b}+z_{b} \partial_{a}+\delta_{a, b}\right), \\
& d \mu\left(\xi_{a, b}^{\prime}-\xi_{b, a}^{\prime}\right)=z_{a} \partial_{b}-z_{b} \partial_{a}, \\
& d \mu\left(\xi_{a, b}-\xi_{a, b}^{\prime \prime}\right)=-i\left(\partial_{a} \partial_{b}+z_{a} z_{b}\right), \\
& d \mu\left(\xi_{a, b}^{\prime}+\xi_{b, a}^{\prime}\right)=\partial_{a} \partial_{b}-z_{a} z_{b} .
\end{aligned}
$$

Let $\mathrm{MU}(2,2)$ denote the inverse image of $U(2,2)$ in $\operatorname{Mp}(4 ; \mathbf{R})$. Then

$$
\nu=\left.\operatorname{det}^{1 / 2} \otimes \mu\right|_{\mathrm{MU}(2,2)}
$$

is a well-defined unitary representation of $\mathrm{U}(2,2)$. There, it agrees with $\mu$ as given in (8), except for the cases $a=b$ in the first line, which become

$$
\begin{aligned}
d \nu\left(\begin{array}{ccc}
i x_{1} & & \\
& \ddots & \\
& & i x_{4}
\end{array}\right) & =\frac{1}{2} d \nu\left\{\sum_{1}^{2} x_{a}\left(\xi_{a, a}+\xi_{a, a}^{\prime \prime}\right)-\sum_{3}^{4} x_{b}\left(\xi_{b, b}+\xi_{b, b}^{\prime \prime}\right)\right\} \\
& =i\left\{\sum_{1}^{2} x_{a}\left(z_{a} \partial_{a}+1\right)-\sum_{3}^{4} x_{b}\left(z_{b} \partial_{b}\right)\right\} .
\end{aligned}
$$

We know [4, Theorem 4.23] that $\mathcal{H}$ is the direct sum of subspaces

$$
\mathcal{K}_{d}=\text { closed linear span of }\left\{\varphi_{n}: n_{1}+n_{2}-n_{3}-n_{4}=d\right\}
$$

and that

$$
\nu=\bigoplus_{d=-\infty}^{\infty} \nu_{d},
$$

where $\nu_{d}$ represents $\mathrm{U}(2,2)$ irreducibly on $\mathcal{K}_{d}$.

In this note, we are concerned with $\left.\nu_{0}\right|_{\mathrm{SU}(2,2)}$. 
3. Restriction to $\mathrm{S}(\mathrm{U}(2) \times \mathrm{U}(2))$. $\mathrm{S}(\mathrm{U}(2) \times \mathrm{U}(2))$ is a maximal compact subgroup of $\mathrm{SU}(2,2)$, and consists of matrices $g=\left(\begin{array}{ll}A & 0 \\ 0 & B\end{array}\right)$ with $A, B \in \mathrm{U}(2)$ and $(\operatorname{det} A)(\operatorname{det} B)=1$. Its center $Z$ consists of those $g$ with $A=e^{i \theta} I_{2}$ and $B=e^{-i \theta} I_{2}$, while its derived group consists of those $g$ with $A, B \in \mathrm{SU}(2)$. The kernel of $\alpha$ : $\mathrm{SU}(2,2) \rightarrow \mathrm{SO}(2,4)$ is just $\left\{ \pm I_{4}\right\}$. Moreover, $\alpha$ maps $\mathrm{S}(\mathrm{U}(2) \times \mathrm{U}(2))$ onto $\mathrm{SO}(2) \times \mathrm{SO}(4), Z$ onto $\mathrm{SO}(2)$, and $\mathrm{SU}(2) \times \mathrm{SU}(2)$ onto $\mathrm{SO}(4)$.

Now the natural action of $\mathrm{SO}(4)$ on $S^{3}$ lifts to $T^{+}\left(S^{3}\right)$ and so to $X$. By [8] and [1], the action of $g \in \mathrm{SO}(4)$ on $\mathcal{K}_{G}$ sends $f$ to $z \mapsto f\left(g^{-1} \circ z\right)$. Hence $\mathcal{H}_{G}$ is a direct sum of subspaces

$$
\left(\mathcal{H}_{G}\right)_{k}=\text { span of }\left\{\left.f\right|_{X}: f \text { homogeneous polynomial of degree } k\right\},
$$

and

$\left.\pi_{G}\right|_{\text {SO(4) }}$ preserves $\left(\mathcal{H}_{G}\right)_{k}$ and acts irreducibly on

it by the $(k+1)^{2}$-dimensional representation ${ }^{k} \otimes \mathrm{O}^{k}$.

Moreover, results of [8] and [1] prove that

$$
\left(\mathcal{H}_{G}\right)_{k} \text { is the } i(k+1) \text {-eigenspace of } d \pi_{G}\left(\begin{array}{cr|c}
0 & 1 & 0_{2,4} \\
-1 & 0 & \\
\hline & 0_{4,2} & 0_{4,4}
\end{array}\right) .
$$

This describes $\left.\pi\right|_{\mathrm{SO}(2) \times \mathrm{SO}(4)}$ and hence $\left.\pi_{1}\right|_{\mathrm{S}(\mathrm{U}(2) \times \mathrm{U}(2))}$.

On the other hand, let $\mathfrak{h}$ be the Cartan subalgebra of $\mathfrak{u}(2,2)$ consisting of the diagonal matrices seen in (10), and let $\varepsilon_{j}(j=1,2,3,4)$ be the linear functional

$$
\left(\begin{array}{lll}
i x_{1} & & \\
& \ddots & \\
& & i x_{4}
\end{array}\right) \mapsto i x_{j} \quad \text { on } \mathfrak{h} \text {. }
$$

We use the simple root system $\stackrel{\alpha_{1}}{\bigcirc}-\stackrel{\alpha_{2}}{\bigcirc}-\stackrel{\alpha_{3}}{\bigcirc}$ for $u(2,2)$ and $\bigcirc^{\alpha_{1}} \oplus \stackrel{\alpha_{3}}{\bigcirc}$ for $\mathfrak{u}(2) \oplus \mathfrak{u}(2)$, where $\alpha_{j}=\varepsilon_{j}-\varepsilon_{j+1}$. Then [9, Lemma 5.3] $\mathcal{K}_{0}$ is a direct sum of subspaces

$$
\mathcal{H}_{r, r}=\operatorname{span} \text { of }\left\{\varphi_{n}: n_{1}+n_{2}=r=n_{3}+n_{4}\right\}
$$

and

$\left.\nu_{0}\right|_{\mathrm{U}(2) \times \mathrm{U}(2)}$ preserves $\mathcal{H}_{r, r}$ and acts irreducibly on it by the representation with highest weight $\left(\varepsilon_{1}+\varepsilon_{2}\right)+r\left(\varepsilon_{1}-\varepsilon_{4}\right)$.

Hence

$$
\begin{aligned}
& \left.\nu_{0}\right|_{\mathrm{SU}(2) \times \mathrm{SU}(2)} \text { acts irreducibly on } \mathcal{K}_{r, r} \text { by the } \\
& (r+1)^{2} \text {-dimensional representation } \mathrm{O}^{\circ} \otimes \mathrm{O}^{\circ},
\end{aligned}
$$


and

$$
\mathcal{H}_{r, r} \text { is the } 2 i(r+1) \text {-eigenspace of } d \nu_{0}\left(\begin{array}{ll}
i I_{2} & 0 \\
0 & -i I_{2}
\end{array}\right) .
$$

Comparing (14) with (18) and (15) with (19), and remembering that

we have

$$
d \alpha\left(\begin{array}{ll}
i I_{2} & 0 \\
0 & -i I_{2}
\end{array}\right)=\left(\begin{array}{lll|l}
0 & & 2 & 0_{2,4} \\
-2 & & 0 & \\
\hline & 0_{4,2} & & 0_{4,4}
\end{array}\right),
$$

LEMMA. $\left.\pi_{1}\right|_{\mathrm{S}(\mathrm{U}(2) \times \mathrm{U}(2))}$ and $\left.\nu_{0}\right|_{\mathrm{S}_{(\mathrm{U}(2) \times \mathrm{U}(2))}}$ are unitarily equivalent.

4. Equivalence of $\pi_{1}$ and $\nu_{0}$. In the simple root system $\left\{\alpha_{1}, \alpha_{2}, \alpha_{3}\right\}$ of $\S 3, \pi_{1}$ and $\nu_{0}$ have the same lowest highest weight $\varepsilon_{1}+\varepsilon_{2}$. Thus [9, Theorem 5.8] gives our result:

THEOREM. The representations $\pi_{1}$ and $\nu_{0}$ of $\mathrm{SU}(2,2)$ are unitarily equivalent.

\section{REFERENCES}

1. R. J. Blattner, $T^{*} S^{n}-\{0$-section $\}$, informal notes, 1975 .

2. V. Guillemin and S. Sternberg, Geometric asymptotics, Math. Surveys, no. 14, Amer. Math. Soc., Providence, R. I., 1977.

3. C. Itzykson, Remarks on boson commutation rules, Comm. Math. Phys. 4 (1967), 92-122.

4. D. Kazhdan, B. Kostant and S. Sternberg, Hamiltonian group actions and dynamical systems of Calogero type, Comm. Pure Appl. Math. 31 (1978), 482-508.

5. B. Kostant, Quantization and unitary representations. I. Prequantization, Lectures in Modern Analysis and Applications. III (C. Taam, Ed.), Lecture Notes in Math., vol. 170, Springer-Verlag, Berlin, 1970, pp. 87-208.

6. G. Mack and I. Todorov, Irreducibility of the ladder representations of $U(2,2)$ when restricted to the Poincare subgroup, J. Mathematical Phys. 10 (1969), 2078-2085.

7. E. Onofri, Dynamical quantization of the Kepler manifold, J. Mathematical Phys. 17 (1976), 401-408.

8. J. H. Rawnsley, A nonunitary pairing of polarizations for the Kepler manifold, Trans. Amer. Math. Soc. (to appear).

9. S. Sternberg and J. A. Wolf, Hermitian Lie algebras and metaplectic representations. I, Trans. Amer. Math. Soc. 238 (1978), 1-43.

10. I. T. Todorov, Discrete series of hermitian representations of the Lie algebra of $U(p, q)$, Lecture notes ICTP, International Centre for Theoretical Physics, Trieste, 1975.

11. J. A. Wolf, Conformal group, quantization, and the Kepler problem, Lecture Notes in Physics, vol. 50, Springer-Verlag, Berlin, 1976, pp. 217-222.

12. Representations associated to minimal co-adjoint orbits, Differential Geometric Methods in Mathematical Physics. II, Lecture Notes in Math., vol. 676, Springer-Verlag, Berlin and New York, 1978, pp. 329-349.

Department of Mathematics, University of California, los Angeles, California 90024

Department of Mathematics, University of California, Berkeley, California 94720 